\title{
Design of Wireless Monitor System Based on ZigBee Technology
}

\author{
Yuan Yuan ${ }^{1, a,{ }^{*}}$, Zhengdong $\mathrm{Li}^{2, \mathrm{~b}}$, Runhua Yan ${ }^{2, \mathrm{c}}$, Lin Wu ${ }^{2, \mathrm{~d}}$, Wei Huang ${ }^{2, \mathrm{e}}$ \\ ${ }^{1}$ College of Electronical and Information Engineering, Beihang University, Beijing, China \\ ${ }^{2}$ Center of Simulation and Training, Chongqing Communication Institute, Chongqing, China \\ ayuan123@126.com, bgusi@foxmail.com, crunhua@foxmail.com, wulin@foxmail.com, \\ ehuangwei@foxmail.com \\ ${ }^{*}$ corresponding author
}

Keywords: wireless; communication ; ZigBee; system; Monitor

Abstract. To solve the traditional problem that the situation in mine is unknown in time, a kind of wireless monitor system based on ZigBee technology is designed. In this system, the security manager can real-time monitor the gas density of multi-location .Compared with traditional system, this system is portable and extensible and it also has advantage of physical size and power consumption. This paper describes both the whole system architecture and the structure of single node. The results show that the system is safe, stable and guarantee the security of miners.

\section{Introduction}

Now, mine safety becomes an important issue. Coal mine operations have been recognized as one of the most risky working environments because of rock falls, the pool equipment and backward management. The underground gas explosion is one of the important reasons of mine accidents. Existing gas detection technology has many limitations, such as the security manager can't real-time monitor the gas density of multi-location. Wireless sensor networks based on the ZigBee technology and gas concentration detection system can effectively solve this problem. Clustering network is combined to detect real-time environmental information such as temperature and humidity, in order to make system more reliable and less energy consumption. The hardware design takes CC2530 as the core of the sensor nodes to store and analyzes the data.

\section{Chip of AD603}

ZigBee is a low power network protocol based on IEEE802.15.4 standard. According to the international standard, ZigBee is a wireless communication technology for short distance and low power. It is also called ZigBee protocol, which is derived from the character of dance of bees. Its characteristic is close, low complexity, self-organizing, low power consumption, low data rate. ZigBee is mainly suitable for automatic control and remote control, which can be embedded in various devices. In short, ZigBee is a cheap, short range wireless network communication technology with lower consumption.

The ZigBee protocol stack achitecture is shown in Figure 1. 


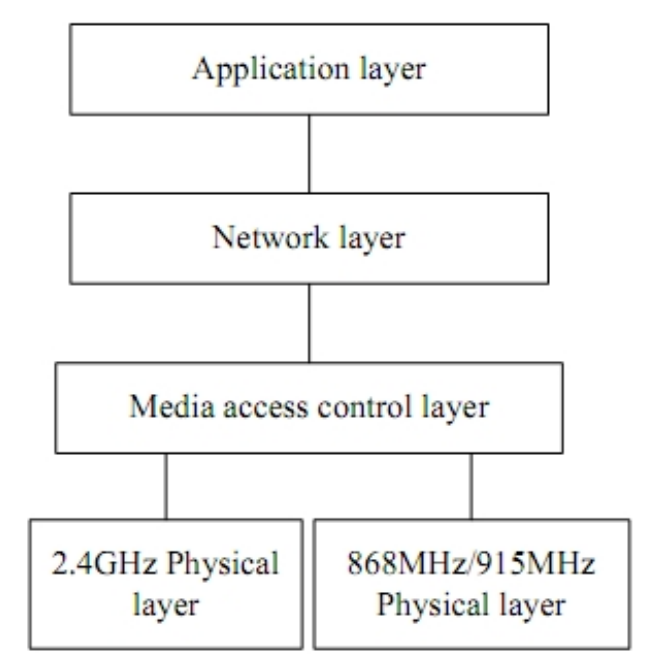

Figure 1. ZigBee protocol stack architecture

\section{Design of the whole system}

The platform consists of multiple gas detection sensor network nodes. Only one node is connected to a computer via USB, we regard this node as root node. Each node is a mobile detection unit. There is a portable gas detector in each node, which can detect the location of the gas concentration. The node can alert according to the gas concentration. The gas concentration data can also be sent to the root node. The root node transmits the gas concentration data to a computer for monitor. The operators can view the gas concentration of mine, and command and manage the system.

The block diagrams of the system are shown in Figure 2.

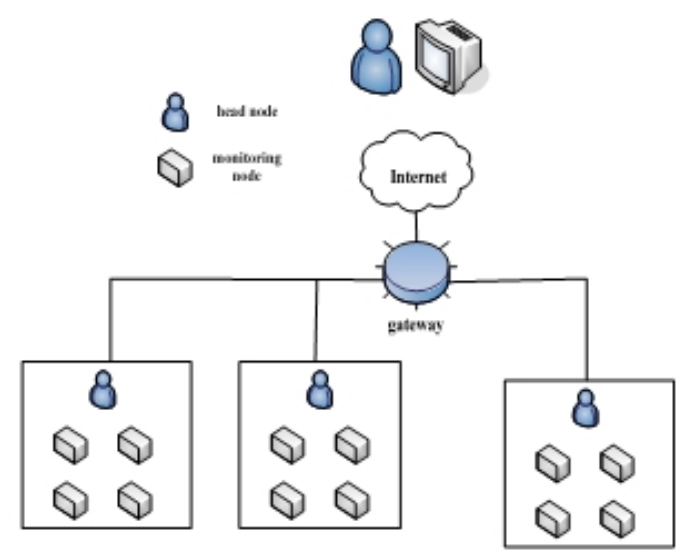

Figure 2. Block diagrams of the whole system

The CC2530 is a true system-on-chip (SoC) solution for IEEE 802.15.4, ZigBee and RF4CE applications. It enables robust network nodes to be built with very low total bill-of-material costs. The CC2530 combines the excellent performance of a leading RF transceiver with an industry-standard enhanced $8051 \mathrm{MCU}$, in-system programmable flash memory, 8-KB RAM, and many other powerful features. The CC2530 comes in four different flash versions: CC2530F32/64/128/256, with $32 / 64 / 128 / 256 \mathrm{~KB}$ of flash memory, respectively. The CC2530 has various operating modes, making it highly suited for systems where ultralow power consumption is required. Short transition times between operating modes further ensure low energy consumption. Combined with the industry-leading and golden-unit-status ZigBee protocol stack ( Z-Stack ${ }^{\mathrm{TM}}$ ) from Texas. Instruments, the CC2530F256 provides a robust and complete ZigBee solution. Combined with the golden-unit-status RemoTI stack from Texas Instruments, the CC2530F64 and higher provide a robust and complete ZigBee RF4CE remote-control solution.

The Structure of the Sensor Node is shown in Figure 3. 


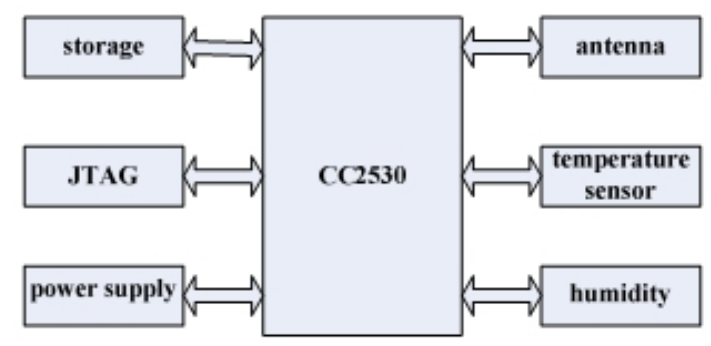

Figure 3. Structure of the Sensor Node

The density of gas is detected by the sensors. According to the sensors, the density of gas is translated into analog voltage which is transmitted to an dual operation amplifier LM2904. The amplified signal and a standard voltage can be used for differential operation.

We chose CC2530 as RF module because it supports IEEE802.15.4 standard, and integrates a 2.4GH RF transceiver of high-performance and 8051 core of low power consumption as a whole. Its peak power output is $4.5 \mathrm{dBm}$, and the biggest communication distance is $60 \mathrm{~m}$. Its good characteristic still comprise low working power, low power consumption and small volume.

The RF circuit is shown as Figure4.

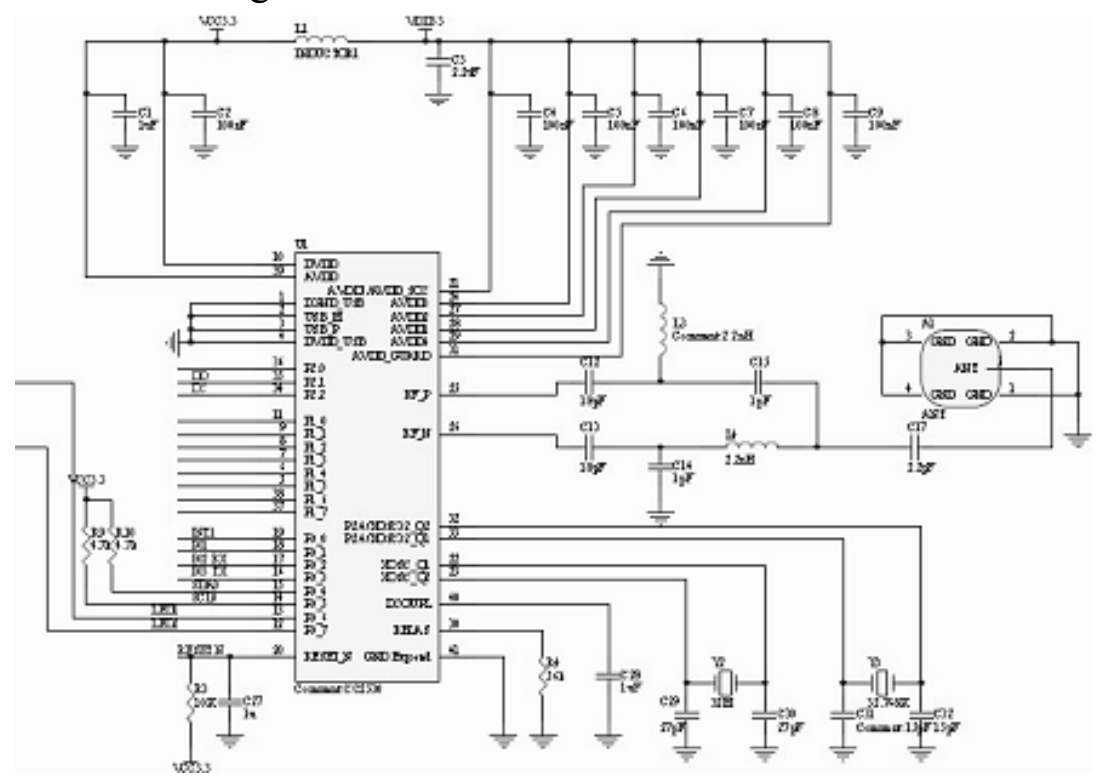

Figure 4. Circuits of operational amplifier

The role of the module of the wireless transceiver is to receive the signals form other nodes and transmit signals to other nodes. In this way, the transceiver realizes the transfer of IEEE802.15.4 in the physical layer.

\section{Process of the system}

Each node can be carried by a worker easily. The data of gas density in the mine will be collected by the node and will be uploaded to the root node. Through the root node, the data of gas density can be transmitted to the computer in the control room. In this way, the workers on the ground can know the gas density near the worker in the mine. In addition, the number of nodes can be added or reduced easily and have nothing to do with the number of workers. Once some nodes are added or quitted, the system would adjust the network automatically.

The monitoring nodes work periodically, and they are awakened in a fixed period. When the data is abnormal, the system would calculate the position based on centroid-weighted localization algorithm, then send position information through the ZigBee. The CC2530 chip has its own determination of RSSI function. The actual measurement from the CC2530 shows RSSI value is in the $0 \times 56 \sim 0 \times 97$. When the distance is too far, the measured RSSI value will become invalid because received signal strength value and distance relations is difficult to obtain steadily due to the influence of 
the environment and multi-path effect. Therefore the position calculation sets that when the received field intensity is less than a certain value, the received smaller field strength value will be not considered. Thus the RSSI values of all the anchor nodes received from the unknown node is analyzed and sorted, and the node positioning calculation uses the first few larger RSSI values.

The monitoring system is combined with the computer. People can easily develop the database management software to collect sensor network upload concentration data. The data of gas density can be recorded in time. The data analysis and scientific warnings can provide adequate conditions and scientific basis for the whole engineering.

\section{Conclusions}

This paper describes a coal mine gas monitoring system based on wireless sensor network technology. Managers on the ground can know the situation of multiple locations of underground gas concentrations. Compared to the current system used in the mines, the system is more susceptible to geographical conditions, and has lower costs and less time-consuming. In addition, the system can be transplanted easily to other operation system. And the system can detect other data, such as humidity, temperature and so on. Therefore the system is a highly practical system which has broad prospects.

\section{References}

[1] The Institute of Electrical and Electronics Engineers, Inc. IEEE Std 802.15.4 ${ }^{\mathrm{TM}}-2003$ [S]

[2] Texas Instrument, CC2530 datasheet[P] 2009.04

[3] Axel Sikora and Voicu F.Groza, "Coexistence of IEEE 802.15.4 with other Systems in the 2.4GHz-ISM-Band," IMTC 2005-Instrumentation and Measurement Technology Conference, Ottawa, Canada, 2005.

[4] Yu Li-min, Li Anqi, Sun Zheng.Design of Monitoring System for Coal Mine Safety Based on Wireless Sensor Network. Mechtronic and Embedded Systems and Applications, 2008. MESA 2008. IEEE/ASME International Conference on 12-15 October 2008:409-414

[5] Qiao Ying-xu, Zhang Zhi-bin, Yang Hong-guo. Application of Wireless Sensor Network in the Monitoring and Control System of Coal Mine Safety. Wireless Communications, Networking and Mobile Computing, 2009. WiCom '09. 5th International Conference on 24-26 September 2009:1-3

[6] Wang Yan, Zheng Ya-ru, Ma Yong.Study on the Coal Mine Personnel Position System Based on Wireless Body Sensor Networks. Medical Devices and Biosensors, 2008. ISSS-MDBS 2008. 5th International Summer School and Symposium on 1-3 June 2008:75-78

[7] Cheng Qiang, Sun Ji-ping, Zhang Zhe, Zhang Fan. ZigBee Based Intelligent Helmet for Coal Miners. Computer Science and Information Engineering, 2009 WRI World Congress on March 31-April 2 2009:433-435

[8] Huang Xu-hui, Zhu Wei, Lu Dong-xin.Underground Miners localization System Based on ZigBee and WebGIS. Geoinformatics, 2010 18th International Conference on 18-20 June 2010:1-5 